\title{
The asymmetric lossy near-perfect lens
}

\author{
S. Anantha Ramakrishna*, J.B. Pendry ${ }^{\dagger}$ \\ The Blackett laboratory, Imperial College, London SW7 2BZ, UK \\ D. Schurig, D.R. Smith ${ }^{\ddagger}$ and S. Schultz
}

Department of Physics, University of California, San Diego, 9500 Gilman Drive, La Jolla, CA 92093-0319, USA

\begin{abstract}
We extend the ideas of the recently proposed perfect lens [J.B. Pendry, Phys. Rev. Lett. 85, $3966(2000)$ ] to an alternative structure. We show that a slab of a medium with negative refractive index bounded by media of different positive refractive index also amplifies evanescent waves and can act as a near-perfect lens. We examine the role of the surface states in the amplification of the evanescent waves. The image resolution obtained by this asymmetric lens is more robust against the effects of absorption in the lens. In particular, we study the case of a slab of silver, which has negative dielectric constant, with air on one side and other media such as glass or GaAs on the other side as an 'asymmetric' lossy near-perfect lens for P-polarized waves. It is found that retardation has an adverse effect on the imaging due to the positive magnetic permeability of silver, but we conclude that subwavelength image resolution is possible inspite of it.
\end{abstract}

\section{INTRODUCTION}

The electromagnetic radiation emitted or scattered by an object consists of a radiative component of propagating modes and a near-field component of non-propagating modes whose amplitudes decay exponentially with distance from the source. For a monochromatic source, the electromagnetic field in free space can be expressed as a Fourier sum over all wave vectors:

$$
E(x, y, z ; t)=\sum_{k_{x}, k_{y}, k_{z}} E\left(k_{x}, k_{y}, k_{z}\right) \exp \left[i\left(k_{x} x+k_{y} y+k_{z} z-\omega t\right)\right]
$$

where $k_{x}^{2}+k_{y}^{2}+k_{z}^{2}=\omega^{2} / c^{2}$ and $c$ is the speed of light in free space. For $k_{x}^{2}+k_{y}^{2}>\omega^{2} / c^{2}, k_{z}$ is seen to be purely imaginary, and the case is similiar for $k_{x}$ and $k_{y}$. The near-field consists of these partial waves with imaginary wave vectors and decays exponentially away from the source. These are the high-frequency Fourier components describing the fast varying spatial features on the object and are never detected in conventional imaging using conventional lenses. This lack of information results in the limitation to conventional imaging that sub-wavelength features of a source cannot be resolved in the image. In order to overcome this limitation, scanning near-field optical microscopy (SNOM) was proposed where the near-field of the radiating object is probed by bringing a tapered fibre tip very close to the object (See Ref. [1] for a recent review). A rule of the thumb is that the near-field produced by a (periodic) feature of spatial extent $d$ will decay exponentially at a rate of $d / 2 \pi$ away from the surface. Hence there is a need to get close to the surface at these resolutions, just in order to detect the evanescent field. Impressive advances have been made in this field, but there still remain several problems in understanding the images, phase contrast mechanisms and associated artifacts.

Now in order to completely reconstruct the object, we would need to amplify these evanescent waves and give the appropriate phase shift for the propagating components. This is precisely what the recently proposed perfect lens with negative refractive index accomplishes [2]. Veselago had noted a long time ago [3] that due to the reversal of Snell's law, a slab of negative refractive index would act as a lens in that the rays from a source on one side would get refocussed on the other side to form an image. But the amplification of evanescent waves by a slab of negative refractive index noted by Pendry [2] was a surprising and new result. The possibility of negative refractive media has already been experimentally demonstrated in the microwave region of the spectrum [0.5]. Both the dielectric constant and the magnetic permeability are negative in a negative refractive medium, and the electromagnetic waves in such a medium will be left-handed as a consequence of Maxwell's equations. No known natural materials have negative magnetic permeability, and the negative refractive media are meta-materials consisting of interlaced periodic arrays

\footnotetext{
*E-mail: s.a.ramakrishna@ic.ac.uk, Tel: +44 020 75947597, FAX: +44 02075947604

${ }^{\dagger}$ E-mail: j.pendry@ic.ac.uk

${ }^{\ddagger}$ E-mail: drs@ucsd.edu
} 
of split ring resonators $[6]$ and thin wires [7]. Pendry also showed that a thin slab of silver with negative dielectric constant would act as near-perfect lens for near-field imaging with P-polarized waves in the electro-static limit, with the resolution limited only by absorption in the lens (silver). More recently, we have examined some consequences of deviations in the dielectric constant and magnetic permeability from the perfect lens conditions $(\epsilon=-1, \mu=-1)$ on the resolution of the lens [8] and found that the restrictions on $\epsilon, \mu$ were quite severe, but achieveable by current day technology. The main advantage of this near-field perfect lens over the current methods of SNOM is that a complete image is generated at the image plane. This would be important in many applications, for example, in-situ imaging of biological molecules and processes by flourescence imaging.

Here we extend the ideas of Pendry's original work. We show that an asymmetric lens consisting of a slab of negative refractive index bounded by media of different positive refractive index can also act as a near-perfect lens. We then examine the nature of the surface modes which are responsible for the amplification of the evanescent waves. In particular, we study the case of a film of silver (with negative dielectric constant) deposited on other media such as glass or GaAs with positive dielectric constant as a near-field imaging lens at optical frequencies. We show that the asymmetry in the system can actually enhance the resolution, depending on the choice of asymmetry and the operating frequency. One of the main advantages of this asymmetric lens is that a very thin metal film deposited on a solid substrate (Glass or GaAs) will be mechanically much more stable than a free-standing metal film. We also study the effects of retardation on the system and conclude that sub-wavelength resolution is possible inspite of the adverse effects of retardation and absorption.

\section{THE ASYMMETRIC SLAB}

[Insert figure 1 about here ]

Typically we consider the asymmetric lens to be a slab of negative refractive medium of thickness $d$, dielectric constant $\epsilon_{2}$ and magnetic permeability $\left(\mu_{2}\right)$ between media of differing refractive indices (See figure 1). We will consider the medium-1 on one side of the source to be air $\left(\epsilon_{1}=1, \mu_{1}=1\right)$ and some other dielectric or magnetic material $\left(\epsilon_{3}, \mu_{3}\right)$ on the other side. We will consider the object plane to be in medium- 1 at a distance $d / 2$ and the image plane, where we detect the image inside medium-3 at a distance $d / 2$ from the edge of the slab. Let P-polarized light be incident on the slab from the medium-1, with the magnetic field" given by

$$
H_{1 p}=\exp \left(i k_{z}^{(1)} z+i k_{x} x-i \omega t\right)
$$

where $k_{x}^{2}+k_{z}^{(j)^{2}}=\epsilon_{j} \mu \omega^{2} / c^{2}(j=1,2,3$ for the different media). We will work in two dimensions for reasons of simplicity. If the index of refraction is negative $\left(\epsilon_{2}<0, \mu_{2}<0\right)$, then the Maxwells equations and causality demand that $k_{z}^{(2)}=-\sqrt{\epsilon_{2} \mu_{2} \omega^{2} / c^{2}-k_{x}^{2}}$ if $k_{x}<\sqrt{\epsilon_{2} \mu_{2}} \omega / c$, and $k_{z}^{(2)}=i \sqrt{k_{x}^{2}-\epsilon_{2} \mu_{2} \omega^{2} / c^{2}}$ if $k_{x}>\sqrt{\epsilon_{2} \mu_{2}} \omega / c$ [2]. The transmission coefficient for the P-polarized light across the slab is

$$
T_{p}\left(k_{x}\right)=\frac{4\left(\frac{k_{z}^{(1)}}{\epsilon_{1}}\right)\left(\frac{k_{z}^{(2)}}{\epsilon_{2}}\right) \exp \left(i k_{z}^{(2)} d\right)}{\left(\frac{k_{z}^{(1)}}{\epsilon_{1}}+\frac{k_{z}^{(2)}}{\epsilon_{2}}\right)\left(\frac{k_{z}^{(2)}}{\epsilon_{2}}+\frac{k_{z}^{(3)}}{\epsilon_{3}}\right)-\left(\frac{k_{z}^{(1)}}{\epsilon_{1}}-\frac{k_{z}^{(2)}}{\epsilon_{2}}\right)\left(\frac{k_{z}^{(3)}}{\epsilon_{3}}-\frac{k_{z}^{(2)}}{\epsilon_{2}}\right) \exp \left(2 i k_{z}^{(2)} d\right)} .
$$

We immediately see that if either $\epsilon_{2}=-\epsilon_{1}$ and $\mu_{2}=-\mu_{1}$, or $\epsilon_{2}=-\epsilon_{3}$ and $\mu_{2}=-\mu_{3}$, then $T_{p}=\left[2 \epsilon_{3} k_{z}^{(1)} /\left(\epsilon_{1} k_{z}^{(3)}+\right.\right.$ $\left.\left.\epsilon_{3} k_{z}^{(1)}\right)\right] \exp \left(-i k_{z}^{(2)} d\right)$ and the amplification of the evanescent waves as well as the phase reversal for the propagating components results. Thus, the system does not have to be symmetric $\left(\epsilon_{1}=\epsilon_{3}, \mu_{1}=\mu_{3}\right)$ as in the original work of Pendry for accomplishing amplification of evanescent waves. In this asymmetric case, however, the field strength at the image plane differs from the object plane by a constant factor, and thus the image intensity is changed. Further, it is easily verified that wave-vectors with different $k_{x}$ will refocus at slightly different positions unless $\epsilon_{3}=\epsilon_{1}$ and $\mu_{3}=\mu_{1}$ (i.e. the symmetric case) or in the limit $k_{x} \rightarrow \infty$. Thus there is no unique (perfect) image plane and we should expect the image to suffer from aberrations. Hence, we term the asymmetric slab a near-perfect lens. A similiar result holds for the S-polarized wave incident on the slab. Again, we point out that with this asymmetric lens, the

\footnotetext{
${ }^{1}$ It is more convenient to use the magnetic field for the P-polarized light. The electric field can be obtained by using the Maxwell's equation $\vec{k}^{(j)} \times \vec{B}=-\omega \epsilon_{j} \vec{E}$.
} 
object is considered to be in medium-1 (usually air) and the image is formed inside medium-3 (mostly considered to be a high index dielectric in subsequent sections of this paper).

In the electrostatic (magnetostatic) approximation $\left(k_{x} \rightarrow \infty\right)$, we have $k_{z}^{(j)} \rightarrow i k_{x}$ and the dependence of the transmission coefficient for the $\mathrm{P}(\mathrm{S})$-polarized field on $\mu(\epsilon)$ is eliminated. The transmission coefficient for the $\mathrm{P}$ polarized wave across the slab is then given by

$$
T_{p}\left(k_{x}\right)=\frac{4 \epsilon_{2} \epsilon_{3} \exp \left(-k_{x} d\right)}{\left(\epsilon_{1}+\epsilon_{2}\right)\left(\epsilon_{2}+\epsilon_{3}\right)-\left(\epsilon_{2}-\epsilon_{1}\right)\left(\epsilon_{2}-\epsilon_{3}\right) \exp \left(-2 k_{x} d\right)},
$$

and the amplification of the evanescent waves depends only on the condition on the dielectric constant $\left(\epsilon_{2}=-\epsilon_{1}\right.$ or $\left.\epsilon_{2}=-\epsilon_{3}\right)$. Such a system can be easily realized as several metals act as a good plasma in some range of optical frequencies in that they can have a large negative real part of the dielectric constant with a comparatively small imaginary part. Typically, we take our system to be a thin film of silver deposited on another medium such as glass, GaAs, Silicon. We note that silver is highly dispersive and by choosing the operating wavelength of light approprately, the dielectric constant $\left(\epsilon_{2}\right)$ of silver can be chosen to be either $-\epsilon_{1}$ or $-\epsilon_{3}$. Note that $\mu=+1$ everywhere for such a system. In the electrostatic limit, we can take the object plane and the image plane to be symmetric about the slab at distance a $d / 2$ from the edge of the slab. To arrive at a simple, though approximate, description of the asymmetric lens, we will take the electrostatic limit in the remainder of this section. We will consider the effects of retardation in Section IV.

The link between the amplification of the evanescent waves to the presence of a surface plasmon mode has already been pointed out [2]. To obtain an insight into the process, let us examine the spatial field variation in our system. First, let us consider the transmission $(T)$ and the reflection $(R)$ from the slab as a sum of partial waves arising from multiple scattering at the interfaces:

$$
\begin{aligned}
T & =t_{21} t_{32} e^{-k_{x} d}+t_{21} r_{32} r_{12} t_{32} e^{-3 k_{x} d}+t_{21} r_{32} r_{12} r_{32} r_{12} t_{32} e^{-5 k_{x} d}+\cdots, \\
& =\frac{t_{21} t_{32} e^{-k_{x} d}}{1-r_{32} r_{12} e^{-2 k_{x} d}}, \\
R & =r_{21}+t_{21} r_{32} t_{12} e^{-2 k_{x} d}+t_{21} r_{32} r_{12} r_{32} t_{12} e^{-4 k_{x} d}+\cdots, \\
& =r_{21}+\frac{t_{21} r_{32} t_{12} e^{-2 k_{x} d}}{1-r_{32} r_{12} e^{-2 k_{x} d}},
\end{aligned}
$$

where $t_{j k}=2 \epsilon_{j} /\left(\epsilon_{k}+\epsilon_{j}\right)$ and $r_{j k}=\left(\epsilon_{j}-\epsilon_{k}\right) /\left(\epsilon_{k}+\epsilon_{j}\right)$ are the partial transmission and reflection Fresnel coefficients for P-polarized light (obtained by matching the tangential components of $E$ and $H$ ) across the interface between Media-(j) and (k) (see figure 1). When the perfect-lens condition at any of the interfaces is satisfied, the partial reflection coefficient $r_{j k}$ as well as the partial transmission coefficient $t_{j k}$ for evanescence across the interface diverges. It should be noted, however, that the S-matrix that relates the incoming and the outgoing wave amplitudes in the scattering process is analytic in the complex (momentum or energy) plane. Hence, the sum of the infinite series is still valid due to the analyticity of the S-matrix, and the reflection and the transmission from the slab are well-defined quantities. The above procedure is entirely equivalent to the usual time-honoured practice of decomposing the fields into a complete set of basis states and matching the field amplitudes at the boundaries as follows. Let the fields in regions $-1,2$, and 3 be given by

$$
\begin{aligned}
& H_{1}=e^{-k_{x} z}+R e^{k_{x} z}, \\
& H_{2}=A e^{-k_{x} z}+B e^{k_{x} z}, \\
& H_{3}=T e^{-k_{x} z}
\end{aligned}
$$

where $R$ and $T$ represent the reflection and transmission coefficients of the slab. Matching the tangential components of $H$ and $E$ at the boundaries, we obtain after some trivial algebra:

$$
\begin{array}{r}
A\left(\epsilon_{2}=-\epsilon_{1}\right)=\left(\frac{\epsilon_{3}-\epsilon_{1}}{\epsilon_{3}+\epsilon_{1}}\right) e^{2 k_{x} d}, \quad A\left(\epsilon_{2}=-\epsilon_{3}\right)=0, \\
B\left(\epsilon_{2}=-\epsilon_{1}\right)=1, \quad B\left(\epsilon_{2}=-\epsilon_{3}\right)=\frac{2 \epsilon_{3}}{\epsilon_{3}+\epsilon_{1}}, \\
T\left(\epsilon_{2}=-\epsilon_{1}\right)=\frac{2 \epsilon_{3}}{\epsilon_{3}+\epsilon_{1}} e^{k_{x} d}, \quad T\left(\epsilon_{2}=-\epsilon_{3}\right)=\frac{2 \epsilon_{3}}{\epsilon_{3}+\epsilon_{1}} e^{k_{x} d}, \\
R\left(\epsilon_{2}=-\epsilon_{1}\right)=\left(\frac{\epsilon_{3}-\epsilon_{1}}{\epsilon_{3}+\epsilon_{1}}\right) e^{+2 k_{x} d}, \quad R\left(\epsilon_{2}=-\epsilon_{3}\right)=\frac{\epsilon_{3}-\epsilon_{1}}{\epsilon_{3}+\epsilon_{1}} .
\end{array}
$$


Note that there are two separate cases, $\epsilon_{2}=-\epsilon_{1}$ on the left side, and $\epsilon_{2}=-\epsilon_{3}$ on the right side in the above equations. [Insert figure 2 about here ]

First, we note that while the transmission is the same in both the cases, the spatial variation of the field is completely different as shown in figure 2. Second, there is a non-zero reflection in both cases, and for $\epsilon_{2}=-\epsilon_{1}$ the reflection is also amplified. The first case of $\epsilon_{2}=-\epsilon_{1}$ is exactly the condition for the excitation of a surface plasmon state at the air-silver interface, where the field is seen to be large and decaying on either side of the interface. The other case of $\epsilon_{2}=-\epsilon_{3}$ corresponds to the excitation of a surface plasmon state at the silver-GaAs interface. In this case only the growing solution within the silver slab is present. It is interesting to note that in the symmetric case of $\epsilon_{1}=\epsilon_{3}$, again only this growing solution within the slab is selectively excited. The reflectivity is also zero for the symmetric slab. To understand the role of the surface modes in the amplification of the evanescent field, we note that the condition $\epsilon_{2}=-\epsilon_{1,3}$ is exactly that for a surface plasmon state to exist on an interface between the semi-infinite negative and semi-infinite positive media. In a finite slab with two surfaces, however, the surface plasmons detune from the resonant frequency, and the detuning is exactly of the right magnitude to ensure that they are excited by incident fields to the correct degree for a focussed image.

The presence of a large reflectivity has serious consequences for the use of a lens for near-field imaging applications as it would disturb the object field. We call the first case of $\epsilon_{2}=-\epsilon_{1}$, where the reflection is also amplified, as the unfavourable configuration, and the second case of $\epsilon_{2}=-\epsilon_{3}$ as the favourable configuration of the asymmetric lens. Obviously it would be most advantageous if the reflectivity were zero. Here we draw an analogy with a conventional lens which in the ideal has perfectly transmitting surfaces, but in practice always produces some stray reflection from the lens surface, but nevertheless still provides an acceptable degree of functionality.

\section{EFFECTS OF ABSORPTION}

Now we will consider the effect of absorption in the slab of the negative index medium on the imaging process. We will work in the electrostatic limit or assume that the perfect lens conditions for the magnetic permeability $\left(\mu_{2}=-\mu_{1}\right.$ or $\mu_{2}=-\mu_{3}$ ) is also satisfied. Pendry [2] had shown that the eventual resolution is limited by absorption in the lens. This absorption can be included in the calculation by adding an imaginary part to the dielectric constant. Pendry had used the dispersion $\epsilon_{2}(\omega)=5.7-9.0^{2}(\hbar \omega)^{-2}+i 0.4$ for silver $(\hbar \omega$ in $\mathrm{eV}$ ) and we will continue to use it here. Put $\epsilon_{2}=-\epsilon_{k}+i \epsilon_{2}^{\prime}$, where $k$ is either 1 or 3 . The expression for the transmission becomes (assuming $\epsilon_{2}^{\prime} \ll \epsilon_{k}$ ),

$$
T_{p}=\frac{-4 \epsilon_{k} \epsilon_{3} \exp \left(-k_{x} d\right)}{ \pm i \epsilon_{2}^{\prime}\left(\epsilon_{1}-\epsilon_{3}\right)-2 \epsilon_{k}\left(\epsilon_{1}+\epsilon_{3}\right) \exp \left(-2 k_{x} d\right)}
$$

with the \pm being chosen according to $k$ is either 1 or 3 respectively. The resolution in Pendry's original case was limited because for large $k_{x}$ the exponential term in the denominator becomes smaller than the other term. Now, we can see that there is definitely an advantage in choosing $\epsilon_{k}$ to be the larger of $\epsilon_{1}$ and $\epsilon_{3}$, in order to make the term containing the exponential to dominate in the denominator. Thus the asymmetry can actually help us better the limit on the resolution set by absorption. Fortunately, this also corresponds to the favourable case of $\epsilon_{2}=-\epsilon_{3}$. Choosing $\epsilon_{k}$ to be the smaller of the two would, in contrast, cause degradation of the resolution. Hence, the sub-wavelength resolution proposed earlier by us as the ratio of the optical wavelength to the linear size of smallest resolved feature [8] works out to be

$$
r e s=\lambda_{0} / \lambda_{\min }=-\frac{\ln \left|\epsilon_{2}^{\prime} / 2 \epsilon_{3}\right| \lambda_{0}}{4 \pi d},
$$

1 (assuming $\epsilon_{3} \gg \epsilon_{2}^{\prime}$ and $\epsilon_{3} \gg \epsilon_{1}$ ) in the favourable configuration.

[Insert figure 3 about here ]

Below we will consider the above effects of absorption on the image of two uniformly illuminated slits (intensity =1) obtained in transmission by the asymmetric slab of silver of thickness $d=40 \mathrm{~nm}$. On the other side, we consider several media of higher dielectric constant. We numerically obtain the image at the image plane (considered to be at $d / 2$ in the electro-static limit) and plot these in figures $3(\mathrm{a}, \mathrm{b})$. In figure $3(\mathrm{a})$, we consider the case where the resolution is enhanced due to the asymmetry in the lens. We take $\epsilon_{2}=-\epsilon_{3}+i \epsilon_{2}^{\prime}$ by tuning to the appropriate frequency (lower, according to the dispersion form), where $\epsilon_{3}>1$ for all the media considered: water $\left(\epsilon_{3}=1.77\right)$, glass $\left(\epsilon_{3}=2.25\right)$, zirconia $\left(\epsilon_{3}=4.6\right)$ and silicon $\left(\epsilon_{3}=14.9\right.$ at $\hbar \omega_{s p}=2 \mathrm{eV}$ corresponding to the surface plasmon being excited at the silver-silicon interface). The level of absorption in silver is taken to be approximately the same at all these frequencies. 
We consider the object to consist of two slits, $20 \mathrm{~nm}$ wide and placed apart by a centre to centre distance of $80 \mathrm{~nm}$. We find that the best contrast is obtained for the case of silicon rather than the symmetric lens. In fact, the resolution initially degrades with increasing $\epsilon_{3}$, but improves drastically for large values of $\epsilon_{3}$. In this case, the two slits are not resolved for the case of glass and zirconia. We obtain a limit of the spatial resolution of about 70nm (centre to centre) for the case of silicon. In figure $3(\mathrm{~b})$, we consider the images obtained for the case $\epsilon_{2}=-\epsilon_{1}+i \epsilon_{2}^{\prime}$, where $\epsilon_{1}=1.0$ for air on one side. This is the unfavourable configuration corresponding to the surface plasmon excitation $\left(\hbar \omega_{s p}=3.48 \mathrm{eV}\right)$ at the air-silver interface. We consider a larger object of two slits of width $30 \mathrm{~nm}$, the centres placed apart by $120 \mathrm{~nm}$ and find that the image resolution gets degraded with increasing $\epsilon_{3}$. For the case of Silicon $\left(\epsilon_{3}=16.4\right.$ at this frequency), the image is just resolved according to the Rayleigh criterion for resolution. However, we will not be particularly interested in this case due to the amplified reflected wave which will severely affect the imaging.

\section{EFFECTS OF RETARDATION}

In the electrostatic(magnetostatic) limit of large $k_{x} \sim k_{z} \sim q_{z}$, there is no effect of changing $\mu(\epsilon)$ for the $\mathrm{P}(\mathrm{S})$ polarization. The deviation from the electrostatic limit caused by the non-zero frequency of the electromagnetic wave would, however, not allow this decoupling. We will now proceed to investigate the effects of retardation caused by a finite frequency of the light. It has already been noted [8] that a mismatch in the $\epsilon$ and $\mu$ from the perfect-lens conditions would always limit the image resolution and also leads to large transmission resonances associated with the excitation of coupled surface modes that could introduce artifacts into the image.

[Insert figure 4 about here ]

Let us first examine the nature of the surface plasmons (for P-polarized incident light) at a single interface between a positive and a negative medium. The condition for the existence of a surface plasmon at the interface is [9]

$$
\frac{k_{z}^{(1)}}{\epsilon_{1}}+\frac{k_{z}^{(2)}}{\epsilon_{2}}=0
$$

which gives the dispersion relation:

$$
k_{x}=\frac{\omega}{c}\left[\frac{\epsilon_{2}\left(\epsilon_{2}-\mu_{2}\right)}{\epsilon_{2}^{2}-1}\right]^{\frac{1}{2}},
$$

where it is assumed $\epsilon_{1}=1$ and $\mu_{1}=1$ for the positive medium. Note that Eq. (16) can be satisfied only for imaginary $k_{z}^{(2)}$, when $\epsilon_{2}$ is negative. The dispersion is plotted in figure 4 , where the causal plasma dispersion forms $\epsilon_{2}=1-\omega_{p}^{2} / \omega^{2}$ and $\mu_{2} \simeq 1-\omega_{m p}^{2} / \omega^{2}$ are assumed for the material of the negative medium [7.6]. This is because a dispersionless $\mu(\omega)<0$ at all $\omega$ would not be physical [10]. We can see that the plasmon dispersion take different forms for $\omega_{m p}>\omega_{p}$ (the upper curve) and $\omega_{m p}<\omega_{p}$ (the lower curve). At large $k_{x}$, the plasmon frequency tends to the electrostatic limit of $\omega_{p} / \sqrt{2}$ from either above $\left(\omega_{m p}>\omega_{p}\right.$ ) or (below $\omega_{m p}<\omega_{p}$ ). At small $k_{x}$, the surface plasmon first appears at the light-line $\left(\omega=c k_{x}\right)$. We note that similiar results have been obtained by Ruppin very recently for the dispersion of the surface plasmon modes in negative refractive media for the case $\omega_{p}>\omega_{m p}$ [11].

[Insert figure 5 about here ]

Now, let us next consider the symmetric lossless slab $\left(\epsilon_{1}=\epsilon_{3}, \mu_{1}=\mu_{3}=1, \epsilon_{2}^{\prime}=0\right)$ and examine the nature of these surface modes. As is well known, the two degenerate surface plasmons at the two interfaces get coupled for a thin slab and gives rise to coupled slab modes: a symmetric and an antisymmetric mode. The condition for these resonances (for P-polarized light) are [9]

$$
\begin{aligned}
& \tanh \left(k_{z}^{(2)} d / 2\right)=-\epsilon_{2} k_{z}^{(1)} / k_{z}^{(2)}, \\
& \operatorname{coth}\left(k_{z}^{(2)} d / 2\right)=-\epsilon_{2} k_{z}^{(1)} / k_{z}^{(2)},
\end{aligned}
$$

\footnotetext{
${ }^{2}$ It should be noted that the Rayleigh criterion, in the strict sense, is applicable for the radiative modes. It states that two separate sources are just resolved when the principal maximum of one image coincides with the first minimum in the diffraction pattern of the other. This results in the ratio of the intensity at the mid-point between the sources to the intensity at the maxima of $\simeq 0.811$. We will use this ratio as the equivalent measure for defining the resolution in our case
} 
where $k_{z}^{(j)}=\sqrt{k_{x}^{2}-\epsilon_{j} \mu_{j} \omega^{2} / c^{2}}$. Using the above conditions, the dispersion relation for the coupled surface modes can be obtained and we show them in figure 4, where again we have assumed the dispersion forms $\epsilon(\omega)=1-\omega_{p}^{2} / \omega^{2}$ and $\mu_{2}=1-\omega_{m p}^{2} / \omega^{2}$. We find that the finite frequency changes the dispersion from the electrostatic limit (where it is given by $\left.\omega=\omega_{p} \sqrt{1 \pm \exp \left(-k_{x} d\right)} / \sqrt{2}\right)$. The dispersion relations are qualitatively different for $\omega_{m p}<\omega_{p}\left(\right.$ or $\left.\mu_{2}\left(\omega_{s}\right)>-1\right)$ and $\omega_{m p}>\omega_{p}$ (or $\mu_{2}\left(\omega_{s}\right)<-1$ ). In the former case, there will be at least two transmission resonances at a given $\omega$ for a deviation of $\epsilon_{2}$ from the perfect lens condition. In fact, for small enough negative deviation $\epsilon_{2}=-1-\delta,(\delta>0$, but small), the first condition can be satisfied for three $k_{x}$, and upto four transmission resonances are possible. In the latter case of $\mu_{2}<-1$, there will be at least one transmitted resonance that can be excited at any $\omega$. For small enough positive deviation $\epsilon_{2}=-1+\delta,\left(\delta>0\right.$, but small), the second condition can be satisfied for two $k_{x}$ and upto three transmission resonances are possible. This becomes clear from figure 5(b) and (d), and the transmission resonances are shown in figure 6(a) and (c). Mathematically, the reason for this behaviour can be explained as follows. For $k_{x} \rightarrow \infty, \omega \rightarrow \omega_{s} \pm \delta$ (where $\delta>0$, and $\rightarrow 0$ ), the \pm sign being chosen depending on whether $\mu_{2}<-1$ or $\mu_{2}>-1$. This causes either the lower or the upper branch respectively to intersect the $\omega=\omega_{s}$ line at some point. Physically, this is because the behaviour of both the symmetric and the antisymmetric modes, at large $k_{x}$, have to tend to the uncoupled plasmon dispersion for a single surface. For positive deviation in $\epsilon_{2}$ and large enough $k_{0} d$, it is possible that no surface mode can be excited in either case, but the required amplification of the evanescent waves also does not result.

[Insert figure 6 about here ]

In figure 6 , we show the transfer function from the object plane to the image plane across a slab of negative dielectric constant for the deviations of the various parameters from the perfect lens condition. In figure 6(a), we show the transmission for $\mu_{2}<1$. Most values of $\epsilon_{2}$ give a single resonance. But for the case of small positive deviation $\left(\epsilon_{2}=-0.99998\right)$, three resonances arise, the latter two occur very close together and not resolved in the graph. We note in this case that even a small amount of absorption removes all traces of the resonances at large $k_{x}$. In the case of the P-polarized light and for $\mu_{2}<1$, the effect of the deviation in $\mu$ is much lesser than that for $\epsilon$ (real or imaginary). figure 6(b) shows the transmission for $\mu_{2}>-1$. There are now at least two peaks, the one which was absent in the other case being very close to $k_{x}=k_{0}$. In figure $6(\mathrm{c})$, we show the transmission for $\mu_{2}=1$. The additional transmission resonances at large $k_{x}$ for small negative deviation in $\epsilon_{2}(=-1.001)$ are clearly visible (expanded and shown in figure 6(d)). Again, we note that non-zero absorption in the slab prevents the divergences at the resonances. But the resonance at $k_{x}$ close to $k_{0}$ is not as strongly damped by absorption.

The transmission resonances will cause the Fourier components at that spatial frequency to be disproportionately represented in the image and will introduce 'noise' in the image. The slab will also act as a 'low-pass' filter for the spatial frequencies because of the deviation (in the real or the imaginary parts of $\epsilon_{2}$ and $\mu_{2}$ ) from the perfect lens condition and limits the image resolution [8]. However, any finite amount of absorption in the slab will not allow the transmission to diverge. When the effects of absorption is included in the calculation, the transmitted intensity at the resonances will be large but finite, and we expect that the effects of the resonances will be softened. But absorption also increases the deviation from the perfect lens conditions and will limit the range of spatial frequencies with effective amplification. Hence absorption by itself would not cause a fundamental improvement in the image resolution.

The problem in using the slab of silver as a perfect-lens, even for P-polarized light, is that the imaging is severely affected by the effects of retardation arising from large deviation in the magnetic permeability ( $\mu=1$ everywhere) from the perfect lens condition $\left(\mu_{2}=-\mu_{3}\right)$. As it happens, this large deviation of $\mu$ severely restricts the range of Fourier components which are effectively amplified in the silver film. In fact, the effect of absorption, other than to soften the effects of the transmission resonances, turns out to be small in comparision. For the case of a lossless (with a very small absorption) symmetric slab with negative dielecric constant $\left(\epsilon_{2}=-1, \mu=1\right)$, the plasmon frequency is $\hbar \omega_{s p}=3.48 \mathrm{eV}$, and $k_{0}=1.77 \times 10^{-2} \mathrm{~nm}^{-1}$. As can be seen in figure $7(\mathrm{a})$, the effects of retardation become severe and the image of a pair of slits is completely swamped by the 'noise' and a large peak develops in-between the images of the slit. The positions of the peaks are also different. However, the presence of absorption softens all the singular behaviour at the resonances and we end up with a good image, in spite of the effects of retardation which is now mainly to reduce the intensity of the image. This is shown in figure $7(\mathrm{~b})$ for the case of the symmetric lossy slab of silver in air. The central lobe is very small now and the positions of the peaks are slightly shifted. Although there is significant distortion of the image, the main features of the image are seen to be unaltered. Thus, absorption has an interesting role of limiting the resolution, and yet being vital for the image formation.

[Insert figure 7 about here ]

Now we will consider the effects of retardation on the asymmetric, lossy, negative dielectric lens, with a positive magnetic permeability $\left(\mu_{2}=+1\right)$. In this case, the dispersion for the surface mode looks like the lower branch in 
figure 5 , but with a different value of $\omega_{s}=\omega_{p} / \sqrt{1+\epsilon_{3}}$. The large $\epsilon_{3}$, however, amplifies the effects of the large deviation of the magnetic permeability ( $\mu=1$ everywhere) from the perfect lens conditions. This drastically reduces the range of Fourier frequencies for which effective amplification is possible. This effect is, however, offset by the beneficial and larger effect caused by a reduced optical frequency $(\hbar \omega=2 \mathrm{eV})$ for the surface plasmon excitation at the silver-silicon interface. Thus, the effects of retardation are much reduced and we are closer to the electrostatic limit in this case. The image obtained for the asymmetric lossy lens with silicon on the image side $\left(k_{0}=1.02 X 10^{-2}\right.$ $\mathrm{nm}^{-1}$ corresponding to $\hbar \omega=2 \mathrm{eV}$ ) is shown in figure $7(\mathrm{c})$. The effects of retardation on the image in this case are seen to be much smaller and the image is not 'noisy', although the intensity is much reduced due to the lower transmission, when compared to the electrostatic case. Note that we are able to resolve sub-wavelength features in this case at about $\lambda / 10$ lengthscale. This should be compared to the case of the symmetric lens where a resolution of only about $\lambda / 4$ is obtained, but at a smaller wavelength.

[Insert figure 8 about here ]

Finally, we explore the possibility that a deviation of $\epsilon_{2}$ from the perfect lens condition may be used to compensate for the deviation of $\mu_{2}$ in order to obtain a better image. We show the transmission across the asymmetric slab in figure 8 for different $\epsilon_{2}$, while keeping $\epsilon_{1}=1$ for air and $\epsilon_{3}=+15$ for silicon fixed. This can again be achieved by tuning the frequency of light and using the material dispersion of silver. We note that the slab mode can be excited by making $\epsilon_{2}$ more negative $\left(=-\epsilon_{3}-\delta\right.$, where $\left.\delta>0\right)$, but the transmission remains finite due to the finite absorption even at the resonant $k_{x}$. The transmission is increased within a finite window of $k_{x}$ for such a deviation, even though the transmission decays much faster at higher values of $k_{x} \gg k_{0}$. This is clearly seen in figure 8 for the cases $\epsilon_{2}(\hbar \omega=1.89 \mathrm{eV})=-17+i 0.4$ and $\epsilon_{2}(\hbar \omega=1.85 \mathrm{eV})=-18+i 0.4$. A deviation of $\epsilon_{2}$ on the other side (making it more positive) just reduces the transmission even further. Thus, we can obtain some extra transmission at lower (but sub-wavelength) spatial frequencies at the cost of reduced transmission at higher spatial frequencies. The enhancement in the image features obtained by such a compensation for the deviation in $\mu$ by changing $\epsilon_{2}$ from the perfect lens condition is shown in figure 7 (c) by the dashed curve. The enhanced resolution is clearly visible.

\section{CONCLUSIONS}

In conclusion, we have shown that a slab of negative refractive index imbedded between two media of different (positive) refractive index also behaves as a near-perfect lens whose performance is limited by only the effects of absorption and retardation. It is only necessary that one of the interfaces satisfies the conditions for the existance of a surface mode at the operating frequency. We have shown that the asymmetry can be used to enhance the resolution of the lens against the effects of absorption in the slab. In particular, we examined the case of a negative dielectric lens consisting of a slab of silver with air on one side and any other dielectric medium on the other. We found that retardation effects due to a finite frequency of light and the mismatch in the magnetic permeability ( $\mu=1$ everywhere) limit the image resolution severely, and also results in the excitation of slab resonances that degrade the performance of the lens. Absorption alleviates the effects of the sharp resonances to some extent, and indeed, is vital to the imaging itself. The reduced frequency of light corresponding to the surface plasmon excitation at the interface between silver and high-dielectric-constant medium can reduce the adverse effects of the retardation drastically. We have shown that sub-wavelength imaging at optical frequencies $(\hbar \omega \simeq 2.0 \mathrm{eV})$ is possible by our asymmetric lens consisting of a slab of silver bounded by air on one side and silicon or GaAs on the other, and we obtain a spatial resolution of about 60-70 nm with this system.

The possibility of having a medium to support a thin film of silver permits the construction of a nanoscopic mechanically rugged lens for optical near-field imaging. It also admits the possibility of an integrated detection system by placing, for example, an array of quantum dots at the image plane inside the GaAs (medium-3). It would also be easier to achieve better surface uniformities, which will eventually limit the performance of the lens. One more advantage is that the operating frequency is reduced from $\hbar \omega=3.48 \mathrm{eV}$ in the UV for the symmetric silver slab in air to $\hbar \omega \simeq 2 \mathrm{eV}$ in the visible for the asymmetric lens. The advantages of this reduction of operating frequency from the UV to the visible for optical instrumentation are obvious. The one comparative disadvantage of the asymmetric lens is a non-zero reflection coefficient which can introduce artifacts in the image. This is because the reflected field can now be multiply scattered between the source and the lens to disturb the object field. This is, however, a problem with the current methods of SNOM as well. It might become necessary to account for the reflection in order to obtain good images. 


\section{ACKNOWLEDGMENTS}

The authors would like to acknowledge the support from DoD/ONR MURI grant N00014-01-1-0803.

[1] Greffet, J.-J., and Carminati, R., 19997, Prog. Surface Science, 56, 133.

[2] Pendry, J. B., 2000, Phys. Rev. Lett., 85, 3966.

[3] Veselago, V. G., 1968, Sov. Phys. Uspekhi, 10, 509.

[4] Smith, D. R., Padilla, W.J., Vier, D.C., Nemat-Nasser, S.C., and Schultz, S., 2000, Phys. Rev. Lett., 84, 4184 (2000).

[5] Shelby, R. A., Smith, D. R., and Schultz, S., 2001, Science, 292, 77.

[6] Pendry, J. B., Holden, A. J., Robbins, D. J., and Steward, W. J., 1999, IEEE trans. MTT, 47, 2075.

[7] Pendry, J. B., Holden, A. J., Stewart, W. J., and Youngs, I., 1996, Phys. Rev. Lett., 76, 4773; Pendry, J. B., Holden, A. J., Robbins, D. J., and Steward, W.J., 1998, J. Phys.: Condens. Matter, 10, 4785.

[8] Smith, D. R., Schurig, D., Rosenbluth, M., Schultz, S., Ramakrishna, S. A., and Pendry, J. B., 2001, Unpublished.

[9] Raether, H., 1988,Surface Plasmons (Berlin: Springer-Verlag) Raether, H., 1980, Excitation of plasmons and interband transitions by electrons (Berlin: Springer-Verlag).

[10] Landau, L. D., and Lifshitz, E. D., 1960 Electrodynamics of continuous media (New York: Pergamon Press).

[11] Ruppin, R., 2000, Phys. Lett. A, 277, 61; Ruppin, R., 2001, J. Phys.: Condens. Matter, $13,1811$. 


\section{Figure captions}

Figure 1: The asymmetric lens system consisting of a slab of negative refractive index bounded by air on one side and any positive medium on the other. The object in medium- 1 at a distance $d / 2$ from the silver surface is focussed inside the medium-3. The partial reflection/transmission coefficients across the boundaries are shown.

Figure 2: Schematic of the field strength distribution for i (a) The asymmetric slab with the surface plasmon excited at the air-silver interface, (b) The asymmetric slab with the surface plasmon excited at the interface between silver and the higher dielectric constant medium, (c) The symmetric slab with air on both sides. The field strength at the image plane is the same as the object plane in this case.

Figure 3: The images (intensity at the image plane in arbitrary units) obtained in the electro-static approximation for the favourable and unfavourable configurations showing the effects of absorption in the slab on the image resolution. The positions of the slits which are imaged are shown by dotted lines. The dielectric constants of the media are shown within the brackets in the legends. (a) Enhancement of the image resolution caused by the asymmetric lens when $\epsilon_{2}=-\epsilon_{3}+i 0.4$, i.e., the favourable configuration. The slits $(20 \mathrm{~nm}$ wide and centre-centre spacing of $80 \mathrm{~nm})$ are just resolved for the symmetric case (air), but are well resolved for large $\epsilon_{3}=14.9$ of Silicon. (b) Degradation of the image resolution with increasing dielectric constant of medium-3, when $\epsilon_{2}=-1+i 0.4$, i.e., the unfavourable configuration. The slits, 30nm wide (larger compared to (a)) with a centre-centre spacing of 120nm (more compared to (a)), are barely resolved for the case of Silicon.

Figure 4: The dispersion for the surface plasmon at an interface between a negative and a positive medium when the effects of dispersion are included. The frequency axis is scaled with respect to the bulk plasmon frequency $\left(\omega_{p}\right)$ and the $k_{x}$ axis is scaled with respect to $k_{p}=w_{p} / c$. The light line $\omega=c k_{x}$ and the $\omega=\omega_{s}=\omega_{p} / \sqrt{2}$ line are given by the dotted lines. The dispersion inside the infinite negative medium, $\omega=c k_{x} / \sqrt{\epsilon_{2} \mu_{2}}$ with $\omega_{m p}=2 \omega_{p}$ is shown by the - - curve (1).

Figure 5: The dispersion for the coupled surface plasmons when the effects of retardation are included. The frequency axis is scaled with respect to the bulk plasmon frequency $\left(\omega_{p}\right)$ and the $k_{x}$ axis is scaled with respect to $k_{p}=w_{p} / c$. The light line $\omega=c k_{x}$ and the $\omega=\omega_{p} / \sqrt{2}$ line are given by the dotted lines. (a) $\mu_{2}=+1.0$, (b) behaviour in (a) at larger $k_{x}$. (c) $\mu=1-\omega_{m p}^{2} / \omega^{2}$ with $\omega_{m p}=2 \omega_{p}$. The dispersion in the infinite negative medium, $\omega=c k_{x} / \sqrt{\epsilon_{2} \mu_{2}}$, is shown by the - - curve. The part of the lower branch with the $\diamond$ symbol shows the non-radiative light modes within the slab. (d) behaviour at larger $k_{x}$ in (c).

Figure 6: The absolute value of the) transmitted field at the image plane $\left|T \exp \left(i k_{z}^{(1)} d\right)\right|$, for the symmetric slab as a function of $k_{x}$, showing the resonances caused by deviations in $\epsilon$ and $\mu$ from the perfect lens conditions. The $\epsilon$ and $\mu$ indicated in the graphs are for the material of the slab. It is assumed $\epsilon=1$ and $\mu=1$ outside the slab. The X-axis is scaled with respect to the free-space propagation vector $k_{0}$.

Figure 7: Effects of retardation on the images. $\mu=+1.0$ everywhere. The thickness of the slab $d=40 \mathrm{~nm}$. The object is a pair of slits of width $20 \mathrm{~nm}$, placed apart by $100 \mathrm{~nm}$ shown by the dotted lines. (a) the symmetric lossless lens. (b) The symmetric lossy silver lens with $(\hbar \omega=3.48 \mathrm{eV})$ (c) The asymmetric lossy silver slab with Silicon as medium-3 $\left[\epsilon_{3}(\hbar \omega=2 \mathrm{eV}) \simeq 15\right]$. The dashed line is for the case when a deviation in $\epsilon_{2}=-18.0+i 0.4$ compensates for the deviation in $\mu$ from the perfect lens conditions.

Figure 8: The absolute value of the transmitted field at the image plane across the asymmetric slab for different $\epsilon_{2}$. $\epsilon_{1}=1.0$ and $\epsilon_{3}=15.0$ are kept fixed. 


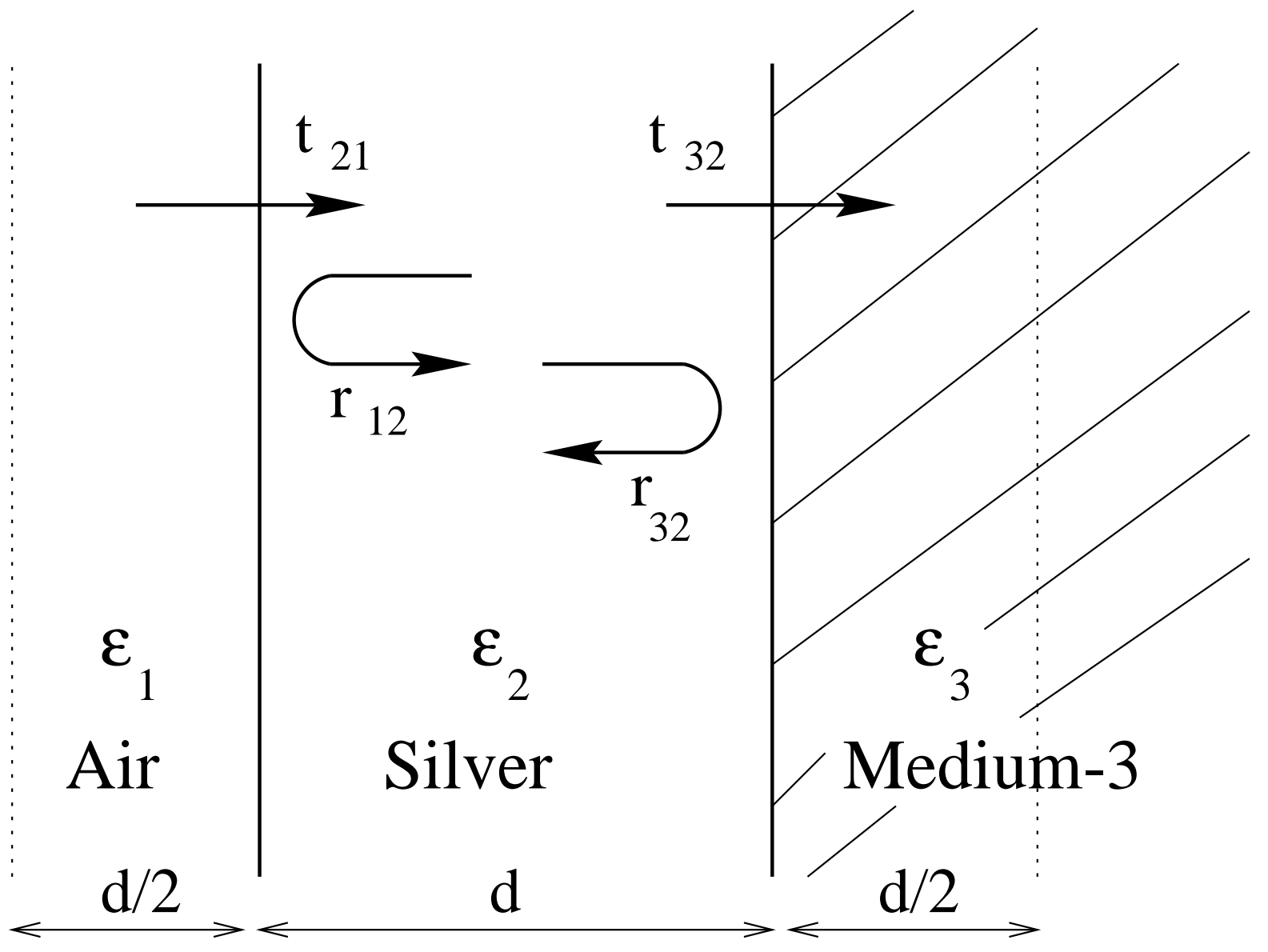




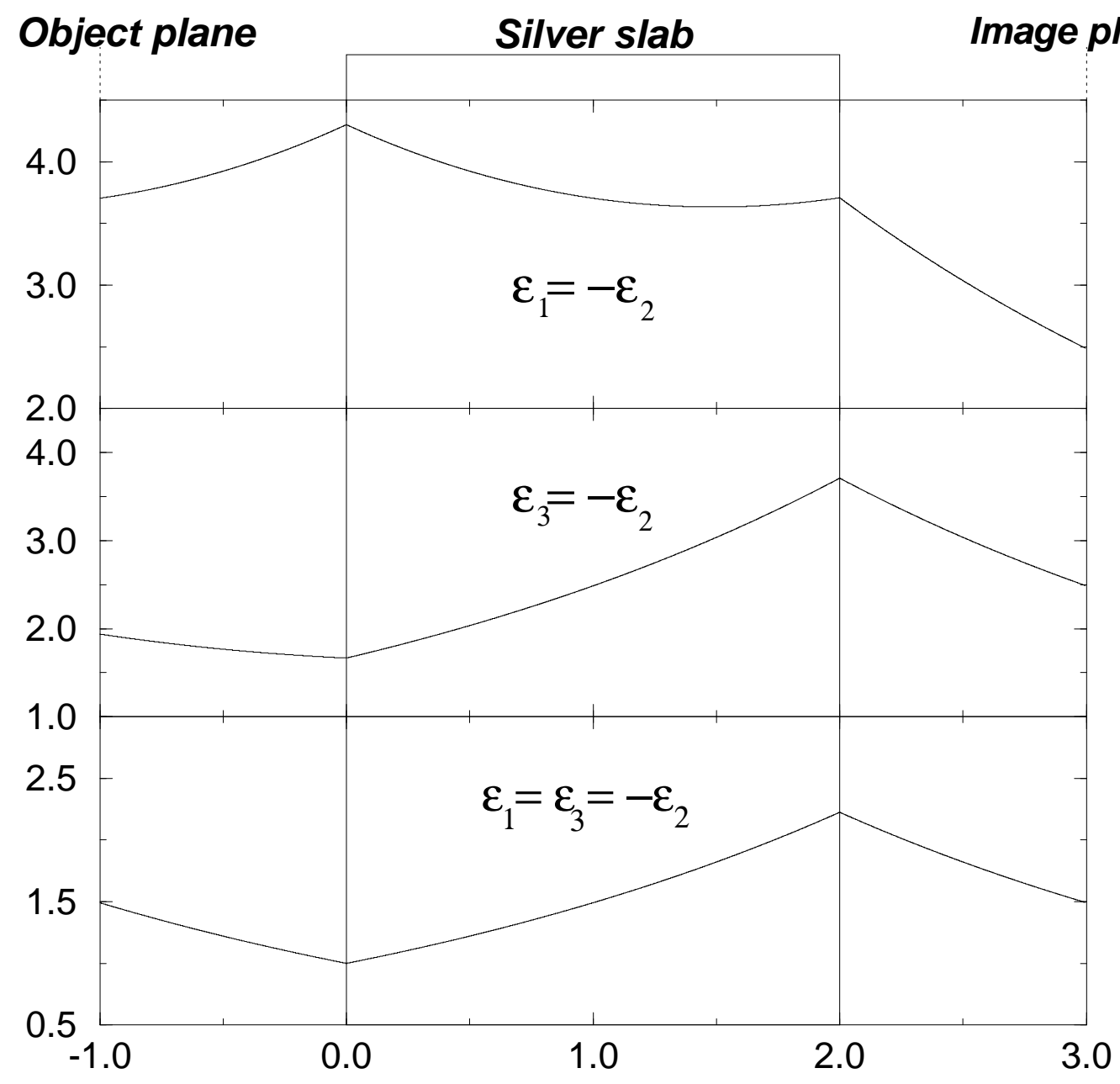



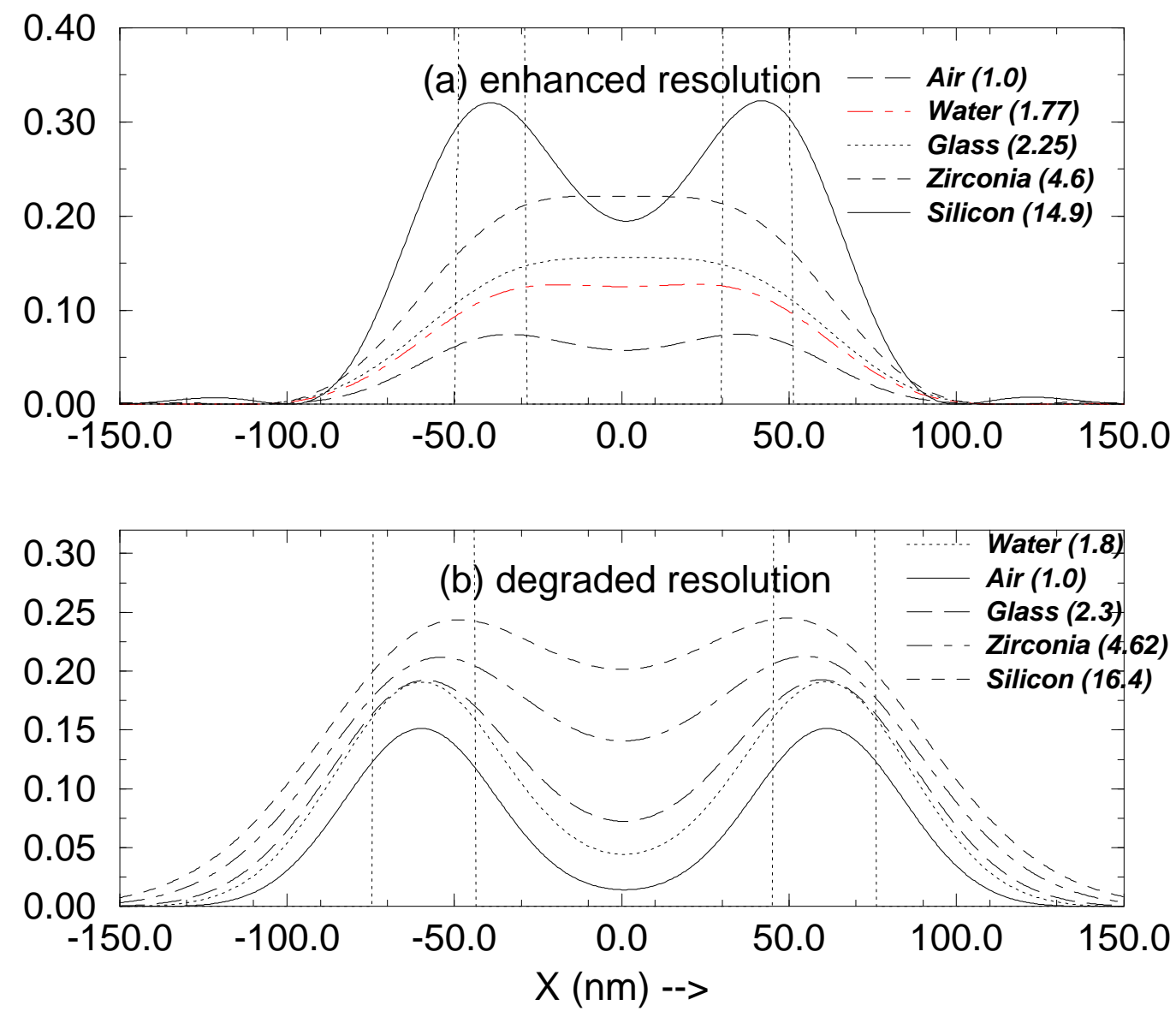


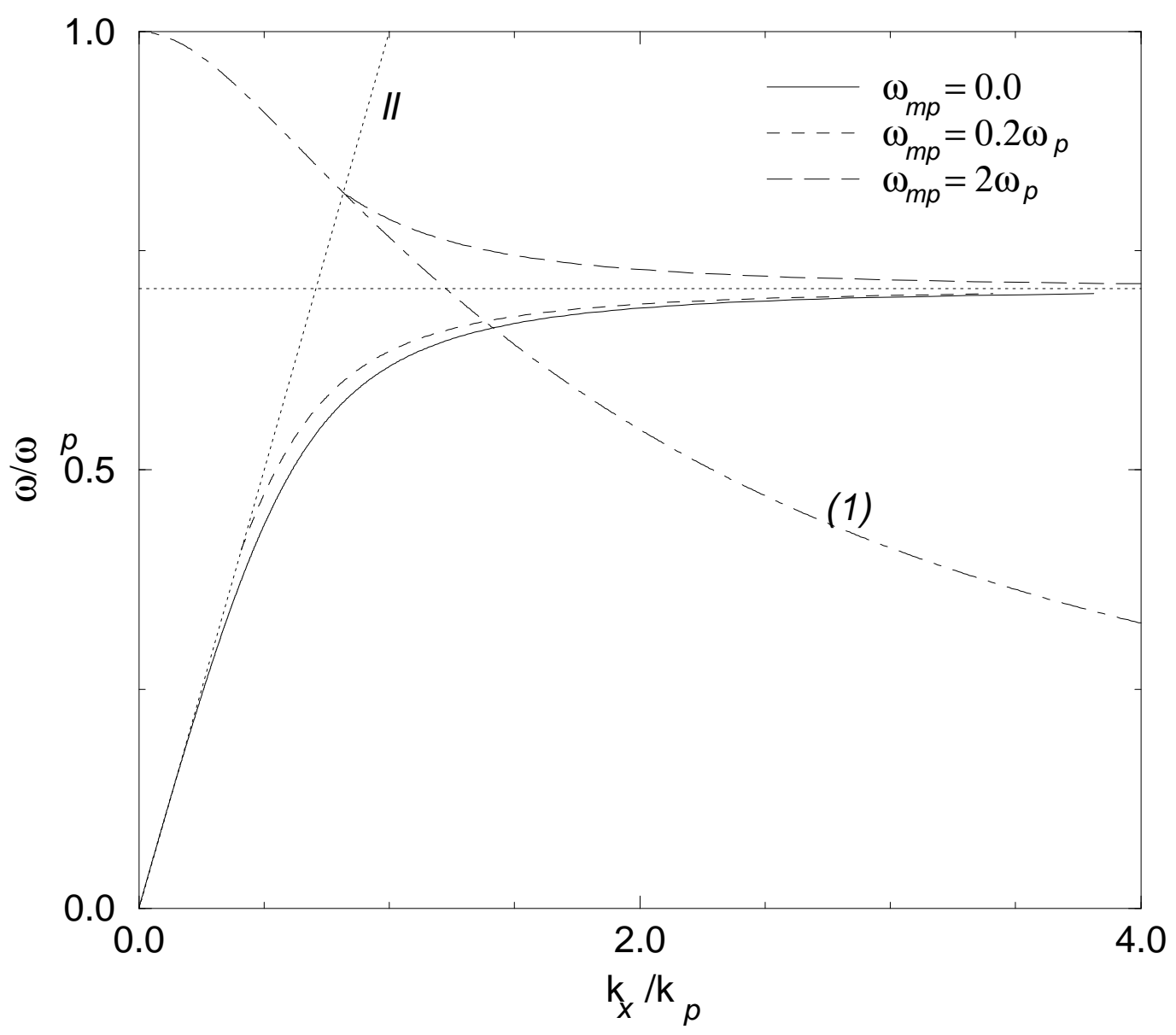



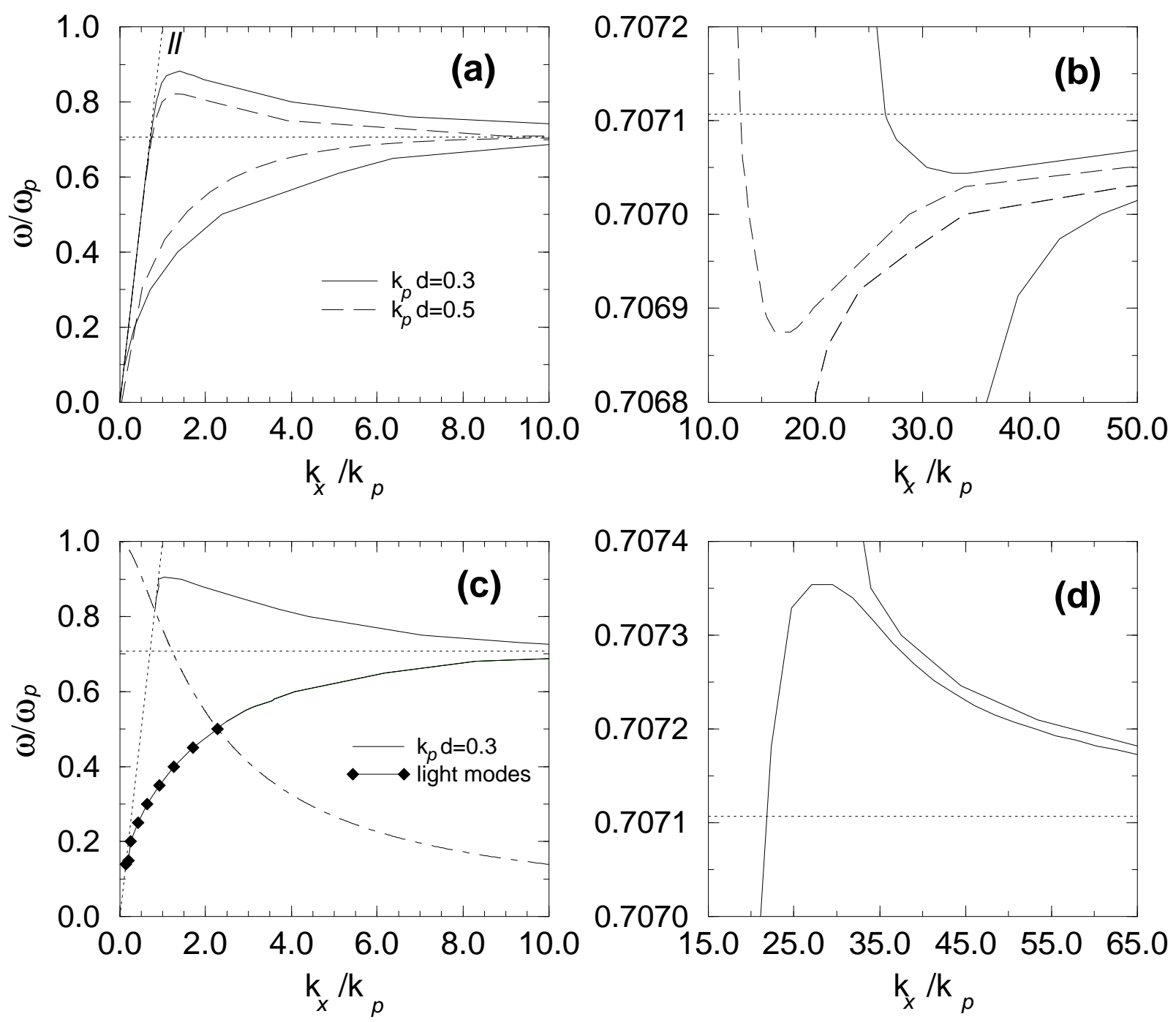


\section{Transmission versus $\mathbf{k}_{x} / \mathbf{k}_{0}$}
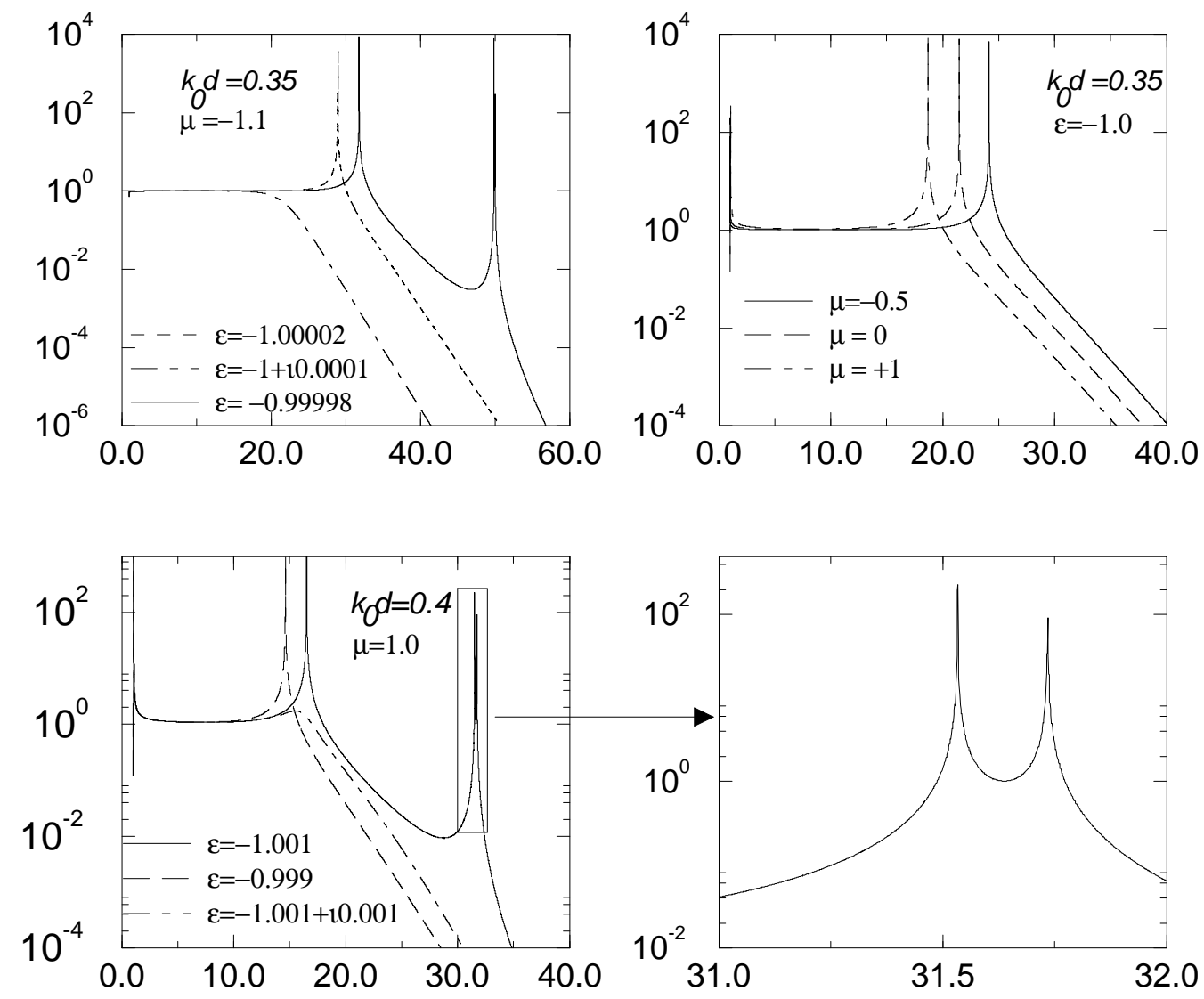
Symmetric lossless lens
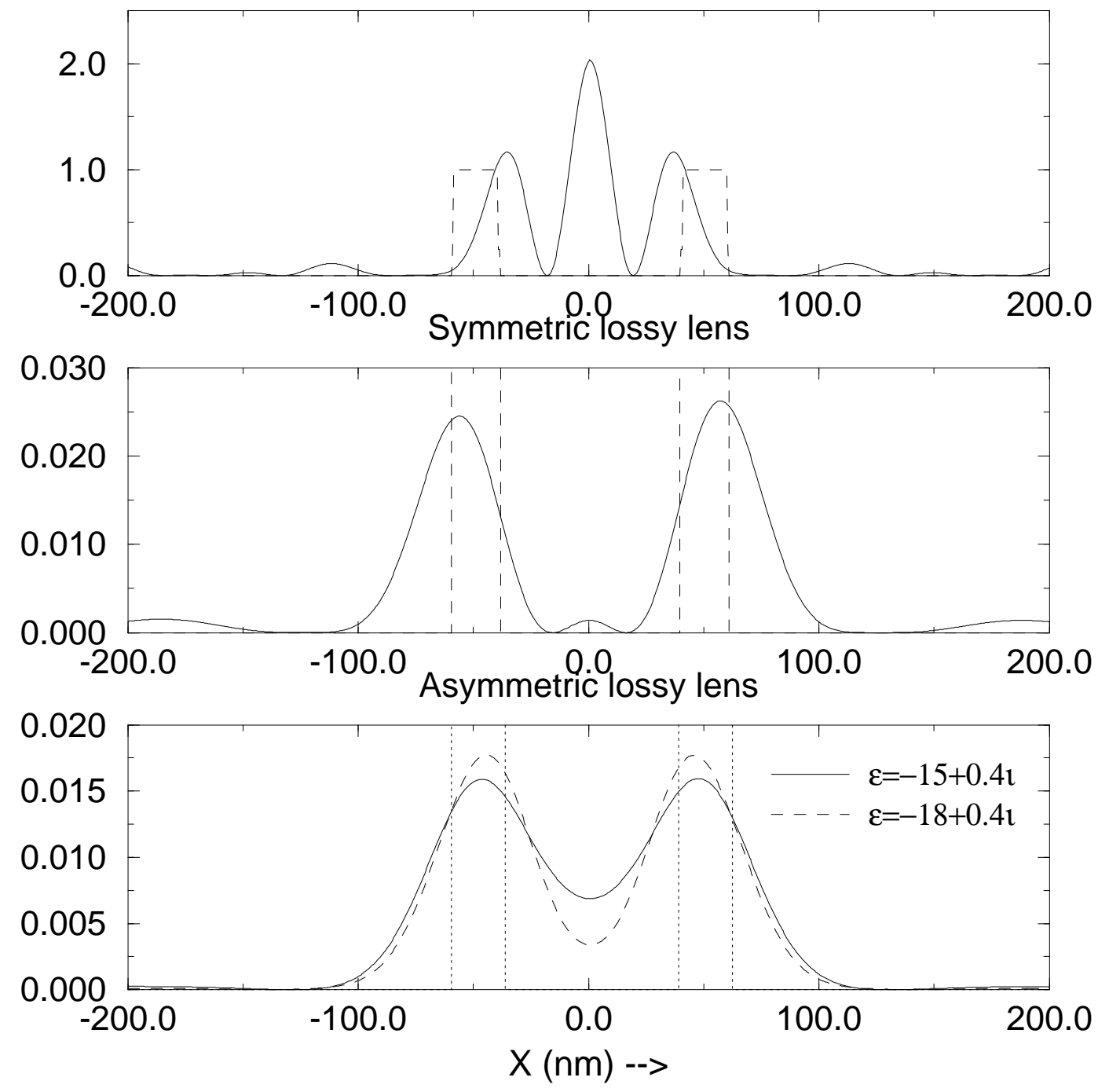
Transfer function for the asymmetric slab

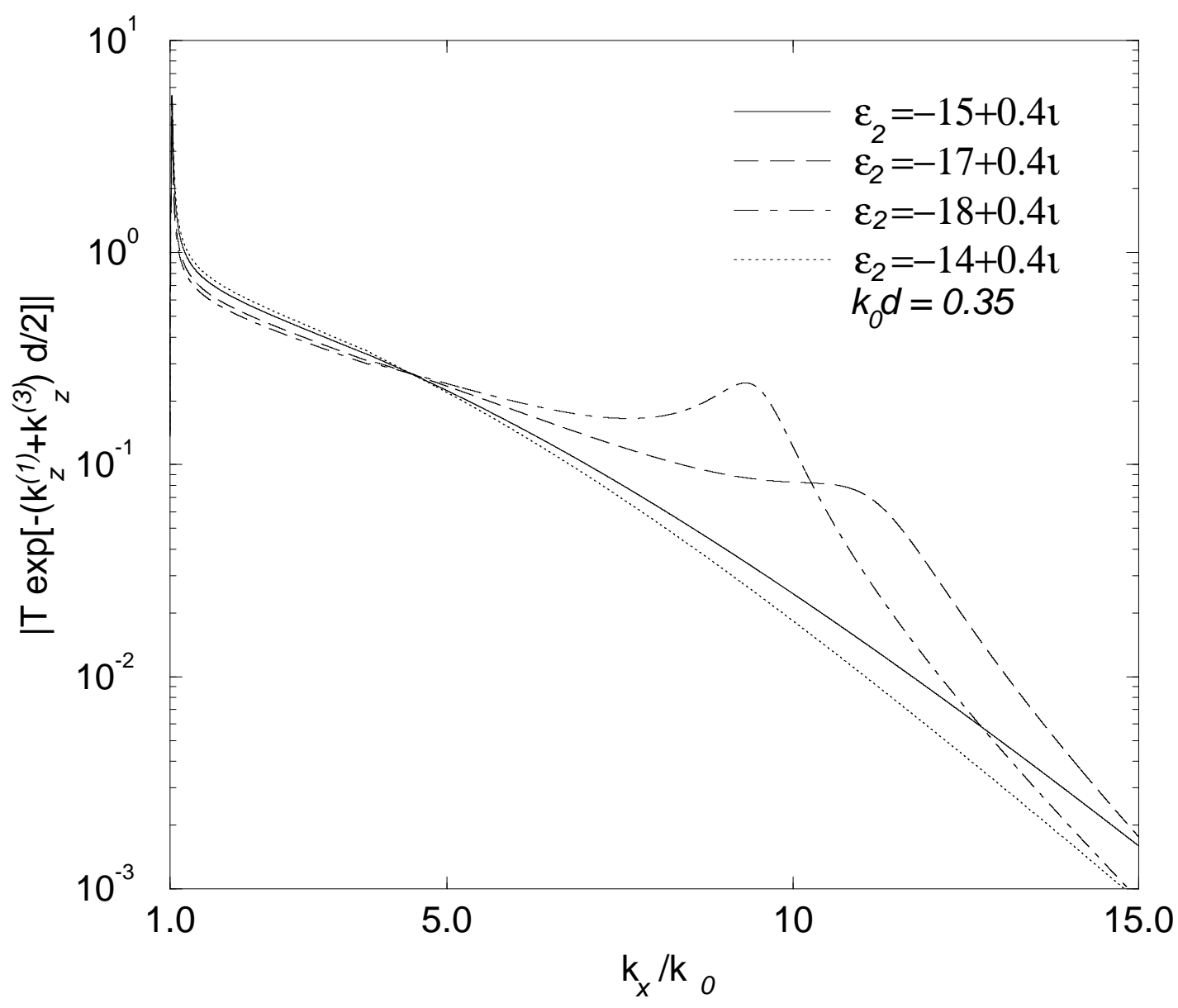

